https://doi.org/10.22319/rmcp.v12i2.5540

Nota de investigación

\title{
Suplementación de ácidos grasos poliinsaturados en el empadre de ovejas nulíparas Katahdin: eficiencia reproductiva y crecimiento pre-destete de las crías
}

\author{
Ricardo Vicente-Pérez $^{\text {a }}$ \\ Ulises Macías-Cruz ${ }^{\mathrm{b} *}$ \\ Leonel Avendaño-Reyes ${ }^{b}$ \\ Enrique O. García-Flores ${ }^{\text {a }}$ \\ Ricardo Martínez-Martínez ${ }^{\text {a }}$ \\ Oziel D. Montañez-Valdez ${ }^{\mathrm{c}}$ \\ José A. Reyes-Gutiérrez ${ }^{\text {c }}$ \\ Alfonso J. Chay-Canul ${ }^{\mathrm{d}}$ \\ María M. Crosby-Galván ${ }^{\mathrm{e}}$
}

a Universidad de Guadalajara. Departamento de Producción Agrícola-CUCSUR, Av. Independencia Nacional 151, C.P. 48900, Autlán, Jalisco, México.

${ }^{\mathrm{b}}$ Universidad Autónoma de Baja California. Instituto de Ciencias Agrícolas, Baja California, México.

${ }^{\mathrm{c}}$ Universidad de Guadalajara. Departamento de Ciencias de la Naturaleza-CUSUR, Jalisco, México.

${ }^{\mathrm{d}}$ Universidad Juárez Autónoma de Tabasco. División Académica de Ciencias Agropecuarias, Villahermosa, Tabasco, México.

${ }^{\text {e }}$ Colegio de Posgraduados. Campus Montecillo, Programa de Ganadería. Estado de México, México.

*Autor de correspondencia: ulisesmacias1988@ hotmail.com 


\section{Resumen:}

Un total de 46 ovejas nulíparas Katahdin se asignaron bajo un diseño de bloques completamente al azar a dos tratamientos dietarios $(n=23)$ alrededor del empadre para evaluar el efecto de adicionar ácidos grasos poliinsaturados (AGP) sobre el comportamiento reproductivo y el crecimiento pre-destete de las crías. Los tratamientos consistieron en dos concentrados isoenergéticos e isoproteícos formulados sin (testigo) y con AGP omega 6, los cuales se ofrecieron durante 42 d (7 d antes y 35 d de exposición al semental). La adición dietaria de AGP disminuyó $(P<0.05)$ el intervalo a estro, sin afectar $(P>0.05)$ las demás variables reproductivas. En crías, el peso al nacimiento no varió $(P>0.05)$ con la adición de AGP; sin embargo, la inclusión dietaria de AGP aumentó $(P<0.05)$ la tasa de crecimiento pre-destete y el peso al destete en corderos, pero no en corderas. La inclusión de AGP en la dieta también mejoró $(P<0.05)$ el crecimiento pre-destete en crías de parto gemelar pero no en crías de parto simple. En conclusión, la inclusión de AGP omega 6 en la dieta de ovejas nulíparas Katahdin durante el empadre, redujo el tiempo de aparición del estro sin afectar otras variables reproductivas; asimismo, mejoró el crecimiento pre-destete de sus crías machos y aquellas nacidas en parto gemelar.

Palabras clave: Ovinos de pelo, Ácido linoleico, Efecto flushing, Fertilidad, Crecimiento post-parto.

Recibido: 09/10/2019

Aceptado: $16 / 07 / 2020$

Las ovejas jóvenes presentan menor eficiencia reproductiva que las ovejas adultas debido a su mayor incidencia de estros silenciosos, cortos y poco intensos ${ }^{(1)}$. Adicionalmente, las crías nacidas de ovejas jóvenes tienden a presentar bajo peso al nacimiento y un crecimiento predestete lento $^{(2)}$. Los ácidos grasos poliinsaturados (AGP) omega 6 (n-6) son esenciales para los rumiantes y favorecen los procesos reproductivos de foliculogénesis, ovulación y actividad estral por estimular la síntesis de estradiol $17 \beta$ a nivel ovárico, así como las prostaglandinas $\mathrm{F} 2{ }_{\alpha}\left(\mathrm{PGF}_{\alpha}\right)$ en el endometrio ${ }^{(3,4)}$. Adicionalmente, los AGP pueden regular procesos epigenéticos y modificar la programación fetal durante la gestación temprana, provocando efectos de largo plazo sobre las crías en su vida post-natal ${ }^{(5)}$. En este sentido, la formulación de la dieta de empadre con AGP n-6 podría ser una estrategia nutricional para mejorar la eficiencia reproductiva y el crecimiento de las crías en ovejas nulíparas.

En ovejas multíparas Pelibuey, la suplementación dietaria de aceite de maíz (rico en AGP n6) mejoró el desarrollo folicular ${ }^{(6)}$, el número de cuerpos lúteos, la calidad de los ovocitos ${ }^{(6)}$ y el desarrollo embrionario ${ }^{(7)}$. Esto se atribuyó a un aumento en las concentraciones séricas de colesterol, insulina, estradiol $17 \beta$ y progesterona ${ }^{(8)}$. Aunque, los efectos de incluir AGP n- 
6 en la dieta de ovejas en empadre sobre sus variables reproductivas siguen siendo poco consistentes a través de los estudios ${ }^{(9,10)}$. Adicionalmente, en ovejas multíparas de lana, la suplementación de AGP n-6 alrededor de la concepción resultó efectiva para reducir la mortalidad pre-destete e incrementar la capacidad de termorregulación post-natal en las crías $^{(11)}$. Cabe mencionar que estos efectos benéficos de los AGP n-6 no se han demostrado en ovejas primíparas de pelo. Por lo tanto, el objetivo del presente estudio fue evaluar el efecto de incluir AGP n-6 en la dieta de empadre sobre la eficiencia reproductiva de ovejas nulíparas Katahdin durante la época reproductiva natural, así como en el crecimiento predestete de sus crías.

El presente estudio se realizó durante la época reproductiva (octubre-noviembre) en el rancho "El Tilzapote", ubicado en la localidad de Ayutita del municipio Autlán de Navarro, Jalisco, México (1948' LN, 104²4' LO y 1,013 msnm). Se utilizaron 46 ovejas nulíparas puras Katahdin, las cuales tenían 9 meses de edad, $43.9 \pm 2.5 \mathrm{~kg}$ de peso vivo (PV) y $3.1 \pm 0.6$ unidades de condición corporal (CC) en una escala de 1 a $5^{(12)}$. Los criterios para seleccionar las ovejas fueron CC entre 2.5 y 3.5 unidades, y presencia de signos de estro un mes previo al inicio del estudio para garantizar ciclicidad ovárica.

Las ovejas se asignaron bajo un diseño de bloques completamente al azar (DBCA; factor de bloque $=\mathrm{PV})$ a uno de dos tratamientos dietarios $(\mathrm{n}=23)$. Los tratamientos consistieron en alimentar a las ovejas con ensilado de maíz sin elote a libre acceso y $400 \mathrm{~g} /$ día/oveja de un concentrado formulado sin (grupo testigo) o con una fuente rica en AGP n-6 (grupo AGP; Cuadro 1).

Cuadro 1: Composición química de los concentrados y del ensilado que se ofrecieron a las ovejas en el empadre

\begin{tabular}{|c|c|c|c|c|c|c|}
\hline \multirow{2}{*}{$\begin{array}{l}\text { Componentes } \\
\text { químicos }\end{array}$} & \multicolumn{2}{|c|}{ Concentrados } & \multirow{2}{*}{ Ensilado } & \multirow{2}{*}{$\begin{array}{l}\text { Ácidos } \\
\text { grasos, \% }\end{array}$} & \multicolumn{2}{|c|}{ Concentrados } \\
\hline & Testigo & AGP & & & Testigo & AGP \\
\hline Proteína cruda, \% & 38.4 & 39.0 & 7.2 & C14:0 & 0.5 & 0.1 \\
\hline Cenizas, \% & 6.0 & 5.0 & 25.7 & $\mathrm{C} 16: 0$ & 20.4 & 12.1 \\
\hline Fibra cruda, $\%$ & 10.2 & 10.1 & 11.6 & $\mathrm{C} 16: 1 \mathrm{n}-7$ & 0.4 & 0.2 \\
\hline FDA, $\%$ & 16.4 & 15.5 & 23.9 & $\mathrm{C} 18: 0$ & 3.3 & 3.6 \\
\hline FDN, $\%$ & 53.0 & 43.5 & 59.1 & C18:1 n-9 & 39.5 & 24.4 \\
\hline TND, $\%$ & 84.0 & 85.0 & 47.4 & C18:2 n-6 & 28.0 & 50.3 \\
\hline $\mathrm{ED}, \mathrm{Mcal} / \mathrm{kg}$ de MS & 3.7 & 3.7 & 2.1 & $\mathrm{C} 20: 0$ & 0.4 & 0.3 \\
\hline \multirow[t]{7}{*}{ EM, Mcal/kg de MS } & 3.0 & 3.1 & 1.7 & $\mathrm{C} 18: 3 \mathrm{n}-6$ & 0.3 & 0.2 \\
\hline & & & & C20:1 n-9 & 0.4 & 0.1 \\
\hline & & & & C18:3 n-3 & 2.8 & 2.4 \\
\hline & & & & $\mathrm{C} 22: 0$ & 0.1 & 0.2 \\
\hline & & & & Poliinsaturados & 31.4 & 53.2 \\
\hline & & & & Monoinsaturados & 40.3 & 24.7 \\
\hline & & & & Saturados & 24.3 & 16.0 \\
\hline
\end{tabular}


Los concentrados fueron isoenergéticos e isoproteicos, pero el concentrado del grupo AGP se formuló con $3 \%$ de aceite vegetal comercial rico en n-6. Para evitar afectaciones en el consumo del concentrado por la consistencia que podría darle el aceite, el concentrado del grupo testigo se formuló usando $3 \%$ de aceite de freiduría, el cual es rico en ácidos grasos oxidados. Las ovejas se alimentaron con las dietas de tratamiento durante 42 días, 7 días antes del empadre y durante los 35 días de empadre. Los concentrados se ofrecieron diariamente en una sola exhibición por las mañanas $(0700$ h), antes de servir el ensilado.

Después del periodo de empadre, las ovejas se alimentaron únicamente con ensilado de maíz hasta cuatro semanas antes del parto. En este último periodo de gestación y durante la lactancia, la alimentación de las ovejas se complementó con 500 g/día/oveja de un concentrado (energía metabolizable $[\mathrm{EM}]=3.0 \mathrm{Mcal} / \mathrm{kg}$ de materia seca $[\mathrm{MS}]$ y proteína cruda $[\mathrm{PC}]=380 \mathrm{~g} / \mathrm{kg}$ de MS), que se formuló con $50 \%$ de pasta de soya, $23 \%$ de canola molida, $19 \%$ de maíz molido, $4 \%$ de minerales, $1 \%$ de urea y $3 \%$ de aceite. Por su parte, las crías tuvieron acceso libre a un alimento iniciador ("creep-feeding") a partir de la segunda semana post-parto hasta el destete (día 90 post-parto), el cual contenía $210 \mathrm{~g}$ de PC/kg de MS y $3.5 \mathrm{Mcal}$ de EM/kg de MS. El alimento iniciador se formuló con $30 \%$ de pasta de soya, $63 \%$ de maíz, $2 \%$ de gluten, $2 \%$ de aceite, $2 \%$ de minerales y $1 \%$ de sustituto de leche.

Durante el experimento, las ovejas se mantuvieron en corrales separados por tratamiento. El espacio en comedero fue suficiente para garantizar que todas consumieran concentrado y ensilado simultáneamente, sin problemas de competencia.

Se colectaron muestras de alimento (ensilado y concentrados) una vez por semana, las cuales fueron usadas para determinar análisis bromatológico (MS, PC, cenizas y fibra cruda) ${ }^{(13)}$ y fracciones de fibra detergente neutro y ácida ${ }^{(14)}$. Mismo procedimiento se realizó para las muestras de ensilado. Los contenidos de $\mathrm{TND}^{(15)}$, energía digestible y $\mathrm{EM}^{(16)}$ fueron calculados con fórmulas. Adicionalmente, el perfil de ácidos grasos se determinó en los concentrados usando un cromatógrafo de gases (HP 6890, USA), el cual estaba equipado con inyector automático (HP 7683, USA), charola con automuestreador y columna capilar de 100 m x $0.25 \mathrm{~mm}$ x $0.2 \mu \mathrm{m}$ (película) a $29 \mathrm{psi}$ (Supelco SP ${ }^{\circledR} 2560$, USA).

Evaluación de estado corporal: El PV y la CC de las ovejas se registró al día 7 antes del empadre, al final del empadre y al parto. Se calculó la ganancia de peso total (GPT) y la ganancia diaria de peso (GDP) de las ovejas durante el periodo que se ofrecieron los tratamientos (42 días).

Evaluación del comportamiento reproductivo: Las ovejas no recibieron ningún tratamiento hormonal antes o durante el empadre por lo que la evaluación de la actividad estral fue acorde al desarrollo de su ciclo estral natural. La actividad estral de las ovejas se registró diariamente (0800 y 1800 h) durante los 35 días del periodo de empadre (del 8 de octubre al 11 de 
noviembre) con ayuda de tres sementales de raza Dorper que tenían fertilidad probada. Los sementales se introdujeron individualmente en los corrales, pero se rotaron entre los tratamientos. Se consideró que una oveja estaba en estro cuando se mostraba receptiva al macho, aceptando la monta natural sin ningún reflejo de movimiento. La fecha y hora de la detección del estro se registró para cada oveja, e igualmente estas hembras en estro se marcaron con un crayón en el dorso y permanecieron en el mismo corral. El número de montas por oveja no se controló, pero se consideró una relación de 15 hembras por macho para asegurar el apareamiento ${ }^{(17)}$. Un mes después de finalizado el periodo de empadre, se realizó el diagnóstico de gestación abdominalmente con ayuda de un equipo de ultrasonido portátil (Modelo DP-10 Vet, Mindray, Shenzhen, China) provisto de un transductor microconvexo. Las ovejas diagnosticadas no gestantes fueron retiradas del corral. Al parto, las ovejas permanecieron en el mismo corral, y solo se registraba fecha de parto y tipo de parto (simple o gemelar).

La información colectada se utilizó para calcular las variables reproductivas. El porcentaje de ovejas en estro se obtuvo expresando el número de ovejas que presentaron estro como un porcentaje del total de ovejas tratadas, y el intervalo a estro contando el número de días transcurridos entre el primer día del empadre y el día que presentó la oveja estro. La tasa de gestación fue el porcentaje de ovejas diagnosticadas gestantes a partir de las ovejas montadas, mientras que la tasa de parición fue el porcentaje de ovejas paridas a partir de las ovejas diagnosticadas gestantes. La fertilidad fue el porcentaje de ovejas paridas a partir del total de ovejas tratadas, y la fecundidad fue el porcentaje de corderos nacidos por oveja montada. La prolificidad se calculó como el número de corderos nacidos por oveja parida. Finalmente, se calculó el porcentaje de ovejas con parto simple o doble a partir del total de ovejas paridas.

Evaluación del crecimiento pre-destete: Todas las crías se identificaron al nacimiento con aretes, y se registró su sexo y peso al nacimiento. Al destete (90 d post-parto), los corderos se pesaron nuevamente para calcular la GPT y la GDP.

Análisis estadístico: Todos los datos se analizaron utilizando el paquete estadístico SAS ${ }^{(18)}$. Las variables de estado corporal, intervalo a estro y prolificidad se sometieron a un análisis de varianza bajo un DBCA. Las variables expresadas en porcentaje se analizaron con una prueba de Ji-cuadrada. El peso al nacimiento y las variables de crecimiento pre-destete de los corderos también se sometieron a un análisis de varianza, pero en un arreglo factorial $2^{3}$ bajo un diseño completamente al azar, donde el modelo consideró los efectos fijos de tratamiento dietario (testigo y AGP), sexo (hembra y macho), tipo de parto (simples y gemelar) y todas las posibles interacciones. En el caso de variables de crecimiento pre-destete se adicionó al modelo el peso al nacimiento como covariable. Las comparaciones de medias se analizaron con una prueba de Tukey a una $\propto=0.05$. La interacción tratamiento x sexo x tipo de parto no fue significativa $(P>0.05)$ por lo cual no se incluyó en los resultados. 
La inclusión dietaria de AGP no afectó ( $P \geq 0.31)$ las variables de estado corporal de las ovejas (PV, CC, GPT y GDP) en ninguno de los tiempos de medición (Cuadro 2). Adicionalmente, la alimentación de las ovejas con AGP redujo $(P=0.05)$ en siete días el intervalo a estro, pero no afectó $(P \geq 0.28)$ las demás variables reproductivas (Cuadro 3). Los resultados de distribución de estros (Figura 1) mostraron que la mayoría $(P<0.05)$ de las ovejas tratadas con AGP presentaron estro en la primera semana de empadre (17.4 vs $47.8 \%$ ), mientras que las ovejas testigo presentaron estro mayormente $(P<0.05)$ en la segunda semana $(43.5 \mathrm{vs}$ $17.4 \%)$. La presencia de estro fue baja para ambos tratamientos en las siguientes tres semanas $(3,4$ y 5$)$, aunque las ovejas testigo presentaron mayor $(P<0.05)$ porcentaje de estro que las ovejas alimentadas con AGP en la cuarta semana.

Cuadro 2: Peso vivo, ganancia de peso y condición corporal de ovejas nulíparas Katahdin alimentadas durante el empadre con un concentrado formulado con (AGP) o sin (testigo) ácidos grasos poliinsaturados n-6

\begin{tabular}{lllll}
\hline & \multicolumn{2}{c}{ Tratamientos } & \multirow{2}{*}{ EE } & \multicolumn{2}{c}{ Valor de } \\
\cline { 2 - 3 } Variables & Testigo & AGP & & \\
\hline Peso vivo, kg & & & & \\
$\quad$ Inicial (7 días pre-empadre) & 44.0 & 43.8 & 0.1 & 0.42 \\
$\quad$ Fin de empadre & 49.5 & 48.6 & 0.6 & 0.31 \\
$\quad$ Parto & 49.5 & 48.7 & 3.7 & 0.84 \\
Ganancia de peso total, kg & 5.6 & 4.8 & 0.6 & 0.37 \\
Ganancia diaria de peso, g/día & 132 & 114 & 14 & 0.37 \\
Condición corporal & & & & \\
$\quad$ Inicial (7 días pre-empadre) & 3.1 & 3.2 & 0.1 & 0.52 \\
$\quad$ Fin de empadre & 3.5 & 3.6 & 0.1 & 0.42 \\
$\quad$ Parto & 3.3 & 3.2 & 0.2 & 0.50 \\
\hline
\end{tabular}

$\mathrm{EE}=$ error estándar.

Cuadro 3: Comportamiento reproductivo de ovejas nulíparas Katahdin alimentadas durante el empadre con un concentrado formulado con (AGP) o sin (testigo) ácidos grasos poliinsaturados $n-6$

\begin{tabular}{|c|c|c|c|}
\hline \multirow[b]{2}{*}{ Variables } & \multicolumn{2}{|l|}{ Tratamiento } & \multirow{2}{*}{$\begin{array}{l}\text { Valor de } \\
P\end{array}$} \\
\hline & Testigo & AGP & \\
\hline Ovejas, $\mathrm{n}$ & 23 & 23 & - \\
\hline Ovejas en estro, $\%$ & $23 / 23(100.0)$ & $22 / 23(95.6)$ & 0.31 \\
\hline Intervalo a estro, días & $17.0 \pm 2.3$ & $10.3 \pm 2.3$ & 0.05 \\
\hline Tasa de gestación, \% & $18 / 23(78.3)$ & $17 / 22(77.3)$ & 0.66 \\
\hline Tasa de parición, \% & $16 / 18(88.9)$ & $16 / 17(94.1)$ & 0.57 \\
\hline Fertilidad, \% & $16 / 23(69.6)$ & $16 / 23(69.6)$ & 1.00 \\
\hline Fecundidad, \% & 24/23 (104.3) & $21 / 23(91.3)$ & 0.35 \\
\hline Partos simples, $\%$ & $8 / 16(50.0)$ & $11 / 16(68.7)$ & 0.28 \\
\hline Partos dobles, $\%$ & $8 / 16(50.0)$ & $5 / 16(31.2)$ & 0.28 \\
\hline Prolificidad, $\mathrm{n}$ & $1.5 \pm 0.1$ & $1.3 \pm 0.1$ & 0.67 \\
\hline
\end{tabular}


Figura 1: Distribución de estros por semana (a) y acumulación de estros semanal (b) durante el periodo de empadre en ovejas Katahdin alimentadas con un concentrado formulado con (AGP) y sin (testigo) ácidos grasos poliinsaturados n-6
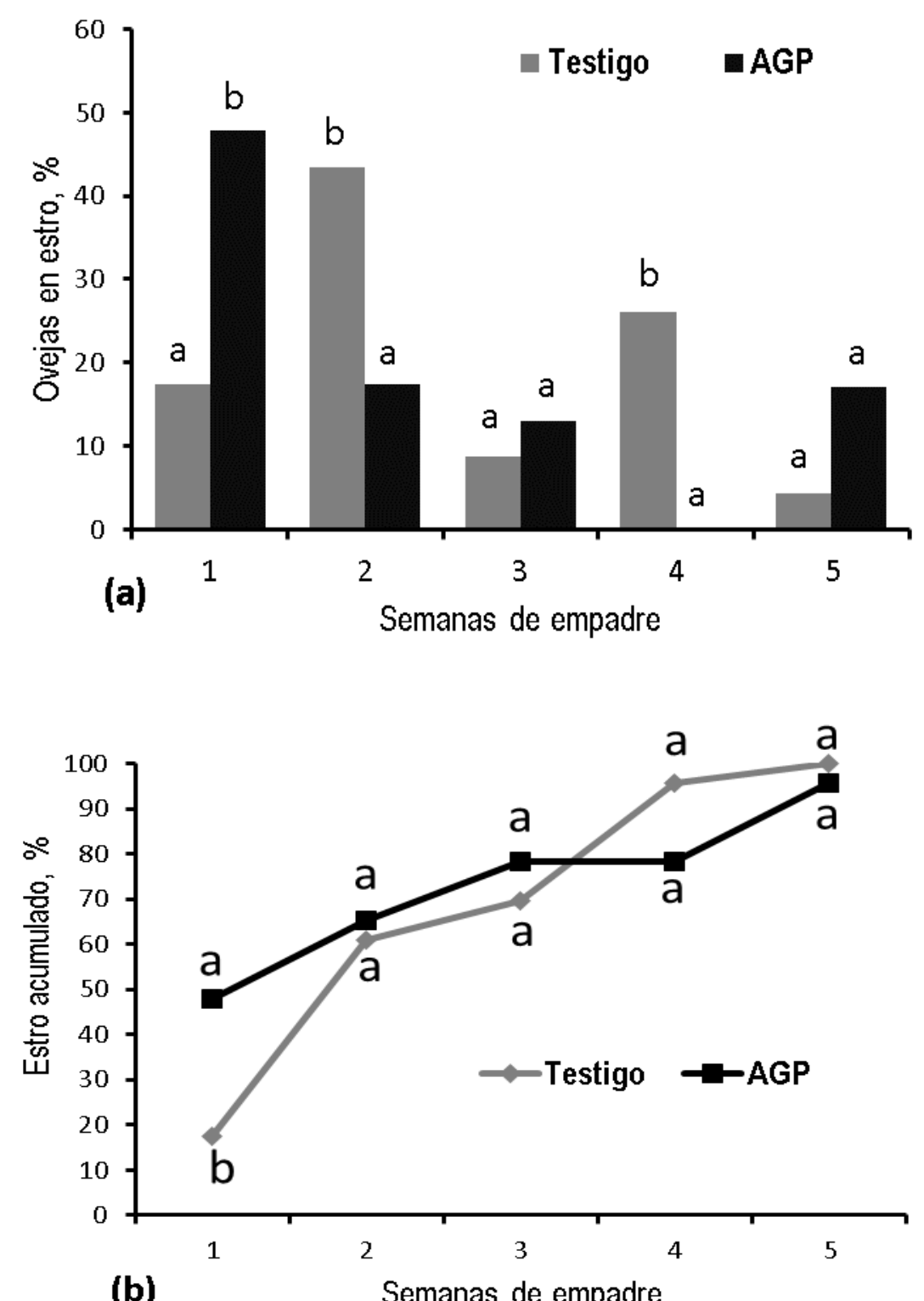

(b)

Semanas de empadre

${ }^{\mathrm{a}, \mathrm{b}}$ Letras diferentes dentro de una misma semana indican diferencias entre tratamientos a $P<0.05$.

Los resultados de crecimiento pre-destete se muestran en el Cuadro 4. La interacción tratamiento x sexo no afectó $(P>0.11)$ el peso al nacimiento, pero sí $(P<0.05)$ la GDP, GPT y peso al destete. El tratamiento de AGP incrementó $(P<0.05)$ la GPT, GDP y peso al destete en corderos, pero no en corderas. Por su parte, la interacción tratamiento $\mathrm{x}$ tipo de parto afectó $(P<0.05)$ el peso al nacimiento y todas las variables del crecimiento pre-destete. Las crías de 
parto simple tuvieron mayor $(P<0.05)$ peso al nacimiento que las crías de parto gemelar en ambos tratamientos dietarios. No obstante, los AGP incrementaron $(P<0.05)$ la GDP, GPT y peso al destete en crías de parto gemelar, pero no $(P>0.05)$ en crías de parto simple. Finalmente, la interacción tipo de parto x sexo afectó $(P<0.05)$ el peso al nacimiento, pero no la GPT, GDP y peso al destete. El peso al nacimiento fue mayor $(P<0.05)$ en crías de parto simple que en crías de parto gemelar en ambos sexos.

Cuadro 4: Crecimiento pre-destete de crías de ovejas Katahdin alimentadas durante el empadre con un concentrado formulado con (AGP) o sin (testigo) ácidos grasos poliinsaturados n-6

\begin{tabular}{|c|c|c|c|c|c|c|}
\hline \multicolumn{2}{|c|}{ Factores de estudio } & \multirow[b]{2}{*}{$\mathbf{n}$} & \multicolumn{4}{|c|}{ Variables de crecimiento } \\
\hline Factor $\mathbf{A}$ & Factor B & & PVN (kg) & PVD (kg) & GPT (kg) & $\begin{array}{l}\text { GDP } \\
\text { (g/día) }\end{array}$ \\
\hline \multicolumn{7}{|c|}{ Interacción tratamiento dietario x sexo } \\
\hline \multirow{2}{*}{ Testigo } & Hembra & 14 & $3.8 \pm 0.2^{\mathrm{a}}$ & $28.0 \pm 1.2^{\mathrm{a}}$ & $24.2 \pm 1.2^{\mathrm{a}}$ & $269 \pm 14^{a}$ \\
\hline & Macho & 10 & $3.9 \pm 0.3^{\mathrm{a}}$ & $28.2 \pm 1.7^{\mathrm{a}}$ & $24.5 \pm 1.7^{\mathrm{a}}$ & $276 \pm 19^{a}$ \\
\hline \multirow{2}{*}{ AGP } & Hembra & 15 & $3.9 \pm 0.2^{\mathrm{a}}$ & $28.5 \pm 1.2^{\mathrm{a}}$ & $24.7 \pm 1.2^{\mathrm{a}}$ & $278 \pm 13^{\mathrm{a}}$ \\
\hline & Macho & 6 & $3.7 \pm 0.3^{\mathrm{a}}$ & $34.3 \pm 2.9^{b}$ & $30.5 \pm 2.9^{b}$ & $341 \pm 33^{b}$ \\
\hline \multicolumn{7}{|c|}{ Interacción tratamiento dietario $\mathrm{x}$ tipo de parto } \\
\hline \multirow{2}{*}{ Testigo } & Simple & 8 & $4.5 \pm 0.3^{\mathrm{a}}$ & $29.6 \pm 2.0^{\mathrm{ab}}$ & $25.9 \pm 2.0^{\mathrm{ab}}$ & $306 \pm 20^{a}$ \\
\hline & Doble & 16 & $3.2 \pm 0.2^{b}$ & $26.6 \pm 1.4^{\mathrm{a}}$ & $22.8 \pm 1.4^{\mathrm{a}}$ & $239 \pm 12^{b}$ \\
\hline \multirow{2}{*}{ AGP } & Simple & 11 & $4.1 \pm 0.2^{\mathrm{a}}$ & $31.6 \pm 2.4^{\mathrm{b}}$ & $27.9 \pm 2.4^{\mathrm{b}}$ & $324 \pm 25^{\mathrm{a}}$ \\
\hline & Doble & 10 & $3.5 \pm 0.2^{b}$ & $31.1 \pm 2.3^{\mathrm{b}}$ & $27.4 \pm 2.3^{\mathrm{b}}$ & $295 \pm 26^{a}$ \\
\hline \multicolumn{7}{|c|}{ Interacción tipo de parto $\mathrm{x}$ sexo } \\
\hline \multirow{2}{*}{ Simple } & Hembra & 14 & $4.5 \pm 0.2^{\mathrm{a}}$ & $29.8 \pm 1.5^{\mathrm{a}}$ & $25.2 \pm 1.5^{\mathrm{a}}$ & $281 \pm 13^{a}$ \\
\hline & Macho & 5 & $4.2 \pm 0.3^{\mathrm{a}}$ & $32.0 \pm 2.7^{\mathrm{a}}$ & $27.9 \pm 2.7^{\mathrm{a}}$ & $310 \pm 29^{a}$ \\
\hline \multirow{2}{*}{ Doble } & Hembra & 15 & $3.2 \pm 0.2^{b}$ & $27.7 \pm 1.5^{\mathrm{a}}$ & $24.5 \pm 1.5^{\mathrm{a}}$ & $272 \pm 16^{\mathrm{a}}$ \\
\hline & Macho & 11 & $3.4 \pm 0.2^{\mathrm{b}}$ & $28.8 \pm 2.3^{a}$ & $25.4 \pm 2.3^{\mathrm{a}}$ & $282 \pm 25^{a}$ \\
\hline
\end{tabular}

$\mathrm{n}=$ número de corderos; $\mathrm{PVN}=$ peso vivo al nacimiento; $\mathrm{PVD}=$ peso vivo al destete; $\mathrm{GPT}=$ ganancia de peso total; GDP= ganancia diaria de peso.

${ }^{\text {ab }}$ Letras diferentes en columnas, dentro de cada interacción doble, indican diferencias $(P<0.05)$.

El estado corporal de las ovejas Katahdin no se modificó por adicionar AGP n-6 en la dieta de empadre, lo cual se esperaba considerando que los concentrados fueron isoenergéticos e isoproteicos, y el nivel de inclusión de aceite en las dietas fue relativamente bajo (3\%). Las dietas en ovinos no deben formularse con más del $6 \%$ de cualquier aceite vegetal, ya que en exceso pueden reducir la actividad microbiana ruminal y, en consecuencia, también el consumo de alimento y estado corporal del animal ${ }^{(3)}$. Los resultados de PV y CC coinciden con lo publicado ${ }^{(10)}$. 
Por otra parte, la inclusión de AGP n-6 en la dieta de empadre acortó en siete días el tiempo de aparición del estro natural en las ovejas Katahdin. Este hallazgo puede ser explicado por dos posibles mecanismos, dependiendo de la etapa del ciclo estral en que se encontraban las ovejas al momento de iniciar su alimentación con la dieta rica en AGP: 1) los AGP causaron una lisis temprana del cuerpo lúteo por estimular la síntesis de PGF2 $\alpha$ al ser precursores del ácido araquidónico ${ }^{(4)}$, y 2) los AGP aumentaron el crecimiento y la capacidad esteroidogénica de los folículos pre-ovulatorios ${ }^{(8)}$. En congruencia con estos resultados, las ovejas Merino también presentaron menor tiempo al estro cuando se alimentaron con semillas de oleaginosas ricas en AGP n-6 durante el periodo de empadre ${ }^{(19)}$.

El uso de AGP n-6 en el periodo de empadre resultó en una estrategia nutricional que ayudó parcialmente a sincronizar naturalmente la actividad estral en las ovejas. Alrededor del $50 \%$ de las ovejas alimentadas con AGP presentaron estro y recibieron monta natural dentro de la primera semana post-inicio del periodo de empadre. Este hallazgo tiene gran relevancia práctica, ya que podría reducir los tiempos de producción asociados con actividades como empadres, partos, cuidados pre-destete de las crías y manejo general del rebaño.

Si bien, el tiempo al estro se redujo por incluir AGP n-6 en la dieta de empadre, el comportamiento reproductivo de las ovejas Katahdin no cambió por la implementación de esta estrategia nutricional. Este hallazgo es contrario a lo esperado. Estudios previos en ovejas Pelibuey indican que la suplementación de AGP n-6 durante el empadre favorece el desarrollo folicular ${ }^{(6)}$ y embrionario ${ }^{(7)}$, así como la tasa ovulatoria ${ }^{(7)}$ y la funcionalidad del cuerpo lúteo $^{(8)}$. Por consiguiente, en este estudio se esperaba mayor tasa de preñez, fertilidad, prolificidad y presencia de partos múltiples en las ovejas nulíparas Katahdin. Contrario a los presentes resultados, las ovejas multíparas Afshari mejoraron su fertilidad, tasa de parición y porcentaje de partos gemelares por recibir AGP n-6 a partir de aceite de girasol protegido con sal de calcio ${ }^{(9,20)}$. En un estudio ${ }^{(17)}$ donde usaron ovejas de los genotipos Pelibuey, Blackbelly y Pelibuey x Blackbelly, se reportó que la suplementación de aceite de soya como fuente de AGP n-6 ayudó a incrementar la prolificidad pero no la tasa de concepción y fertilidad.

Posiblemente, los AGP no mejoraron el comportamiento reproductivo de las ovejas Katahdín porque el tiempo de alimentación con AGP antes del periodo del empadre fue corto (7 días); se requiere ofrecer el concentrado al menos 20 días antes de exponer a las ovejas al macho, y continuar durante el periodo de empadre ${ }^{(9,17,20)}$. Otra posible causa es una baja biodisponibilidad de los AGP en la circulación después de su consumo, considerando que solo el $15 \%$ de ellos llegan intactos al intestino para su absorción, y el resto, es saturado durante la biohidrogenación ruminal ${ }^{(3)}$. Al respecto, Asgari Safdar et al ${ }^{(20)}$ encontraron mayor crecimiento folicular, concentración sérica de hormonas esteroidales, fertilidad, prolificidad y tasa de parición cuando las ovejas consumieron aceite de girasol protegido en sal de calcio comparado a cuando dicho aceite no estaba protegido. 
Por otra parte, el efecto de alimentar ovejas con AGP en el empadre sobre el peso al nacimiento de las crías es contradictorio en la literatura; mientras que algunos estudios no reportan efectos ${ }^{(19)}$, otros indican una mejora en el peso al nacimiento ${ }^{(9,20)}$. En este estudio, la alimentación de las ovejas en el empadre y el sexo de las crías no afectaron el peso al nacimiento, pero el tipo de parto mostró ser un factor importante para definir la variación de dicha variable. Las crías de cualquier sexo u origen de madre fueron más pesadas cuando nacieron en parto simple que en parto gemelar. En la literatura se señala consistentemente esta diferencia de peso al nacimiento debido al tipo de parto ${ }^{(21)}$.

Las crías de ovejas alimentadas con AGP en el empadre mostraron un mejor crecimiento predestete, particularmente, las crías que nacieron macho o en parto gemelar. Actualmente, se conoce que la programación fetal se inicia después de la concepción, y varía entre individuos de acuerdo al sexo y tipo de parto ${ }^{(2)}$. Mientras que los fetos machos se programan para tener un mejor desarrollo muscular postnatal que las hembras ${ }^{(22)}$, los fetos de gestación múltiple a diferencia de los fetos de gestación simple se programan para presentar de manera natural restricción intrauterina en su crecimiento fetal pudiendo afectar negativamente su desarrollo postnatal $^{(23)}$. Por lo tanto, los hallazgos encontrados sugieren que, en ovejas Katahdin, los AGP n-6 en el empadre ejercen efectos benéficos de largo plazo sobre el crecimiento predestete de los corderos a través de modificar su programación fetal en la etapa temprana de la gestación. Adicionalmente, esta estrategia nutricional parece revertir parcialmente la programación fetal natural del crecimiento que presentan los fetos de preñez gemelar; este hecho explica el mejorado crecimiento pre-destete que tuvieron los corderos de origen gemelar nacidos a partir de ovejas alimentadas con AGP. Los AGP se asocian a la programación fetal del metabolismo lipídico y muscular, así como a la conducta y vigor de los neonatos ${ }^{(5)}$. Cabe mencionar que esto se requiere comprobar en estudios futuros donde la programación fetal sea evaluada.

La alimentación con AGP n-6 en el empadre redujo el tiempo de aparición del estro, pero no mejoró el comportamiento reproductivo general en ovejas nulíparas Katahdin durante la época reproductiva natural. Adicionalmente, esta estrategia nutricional tuvo efectos benéficos de largo plazo sobre el crecimiento pre-destete de los corderos y las crías nacidas en parto gemelar.

\section{Agradecimientos}

Se agradece al M.V.Z. Ramón Andrade Mancilla por facilitar su rancho "El Tilzapote”, y por todo el apoyo técnico que brindo durante la realización del experimento. 


\section{Literatura citada:}

1. Corner RA, Mulvaney FJ, Morris ST, West DM, Morel PCH, Kenyon PR. A comparison of the reproductive performance of ewe lambs and mature ewes. Small Ruminant Res 2013;114:126-133.

2. Kenyon PR, Blair HT. Foetal programming in sheep - Effects on production. Small Ruminant Res 2014;118:16-30.

3. Hess BW, Moss GE, Rule DC. A decade of developments in the area of fat supplementation research with beef and sheep. J Anim Sci 2008;86(14 Suppl):E188204.

4. Whates DC, Abayasekara DRE, Aitken RJ. Polyunsaturated fatty acids in males and female reproduction. Biol Reprod 2007;77:190-201.

5. Kabaran S, Besler HT. Do fatty acids affect fetal programing? J Health Popul Nutr 2015;33(14):1-9.

6. Meza-Villalvazo V, Magaña H, Sandoval C, Morales M, Chay A, Trejo A. Efecto de los ácidos grasos poliinsaturados sobre población folicular y calidad ovocitaria en ovejas Pelibuey. Universidad y Ciencia 2013;29:255-261.

7. Herrera-Camacho J, Aké-López JR, Ku-Vera JC, Williams GL, Quintal-Franco JA. Respuesta ovulatoria, estado de desarrollo y calidad de embriones de ovejas Pelibuey superovuladas suplementadas con ácidos grasos poliinsaturados. Téc Pecu Méx 2008;46:107-117.

8. Meza-Villalvazo VM, Magaña-Sevilla H, Rojas-Marquez CA, Sandoval-Castro C, TrejoCordova A. El aceite de maíz incrementa los niveles séricos de progesterona y estradiol en ovejas de pelo. Ecosist Recur Agropec 2018;5:583-589.

9. Daghigh Kia H, Asgari Safdar AH. Effects of calcium salts of fatty acids (CSFA) with different profiles (w-3 and w-6) during the flushing period on reproductive performance on Afshari ewes. Small Ruminant Res 2015;126:1-8.

10. Mirzaei-Alamouti H, Mohammadi Z, Shahir MH, Vazirigohar M, Mansouryar M. Effects of short-term feeding of different sources of fatty acids in pre-mating diets on reproductive performance and blood metabolites of fat-tailed Iranian Afshari ewes. Theriogenology 2018;113:85-91.

11. Clayton EH, Wilkins JF, Friend MA. Intergenerational effects of omega-6 fatty acids. 2. Preliminary evidences for the influences of diet fed to dam at conception on sex ratio of lambs born to their daugthers. Anim Reprod Sci 2017;57:51-59. 
12. Russel AJF, Doney JM, Gunn RJ. Subjective assessment of body fat in live sheep. J Agr Sci 1969;72(3):451-454.

13. AOAC, 2003. Official methods of analysis. 17th Edition current through 2nd Revition.ED. Association of Official Analytical Chemists, Washington DC, USA.

14. Van Soest PJ, Robertson JB, Lewis BA. Methods for dietary fiber, neutral detergent fiber, and nonstarch polysaccharides in relation to animal nutrition. $J$ Dairy Sci 1991;74(10):223-232.

15. Alves AR, Beelen PMG, de Medeiros AN, Neto SG, Beelen RN. Consumo e digestibilidad do feno de sabia por caprinos e ovinos suplementados com polietilenoglicol. Rev Caatinga 2011;24:152-157.

16. NRC. Nutrient requirements of sheep. National Academy Press, Washington, DC. 1985.

17. Cansino-Arroyo G, Herrera-Camacho, Aké-López. Tasas de concepción, fertilidad y prolificidad en ovejas de pelo alimentadas con dietas enriquecidas con ácidos grasos poliinsaturados. Universidad y Ciencia 2009;25:181-185.

18. SAS Institute, SAS/STAT. User's guide statistical released 9.1. 2nd Ed. SAS Intitute, Inc. Cary, NC, USA; 2004.

19. Clayton EH, Friend MA, Wilkins JF. Increasing the proportion of female lambs by feeding Merino ewes a diet high in omega-6 fatty acids around mating. Anim Prod Sci 2016;56(7):1174-1184.

20. Asgari Safdar AH, Sadeghi AA, Chamani M. Effects of different fat sources (saturated and unsaturated) on reproductive performance and biological indices of ewes during flushing period. Trop Anim Health Prod 2017;49:1447-1453.

21. Macías-Cruz U, Álvarez-Valenzuela FD, Olguín-Arredondo HA, Molina-Ramírez L, Avendaño-Reyes L. Ovejas Pelibuey sincronizadas con progestágenos y apareadas con machos de raza Dorper y Katahdin bajo condiciones estabuladas: producción de la oveja y crecimiento de los corderos durante el período pre-destete. Arch Med Vet 2012;44:2937.

22. Sinclair KD, Allegrucci C, Singh R, Gardner DS, Sebastian S, Bispham J, et al. DNA methylation, insulin resistance, and blood pressure in offspring determined by maternal periconceptional B vitamin and methionine status. Proc Natl Acad Sci U S A 2007;104:19351-19356.

23. Van der Linden DS, Sciascia Q, Sales F, McCoard SA. Placental nutrient transport is affected by pregnancy rank in sheep. J Anim Sci 2013;91:644-653. 